\title{
Professor Howard Morris, IFCC President (1946-2019)
}

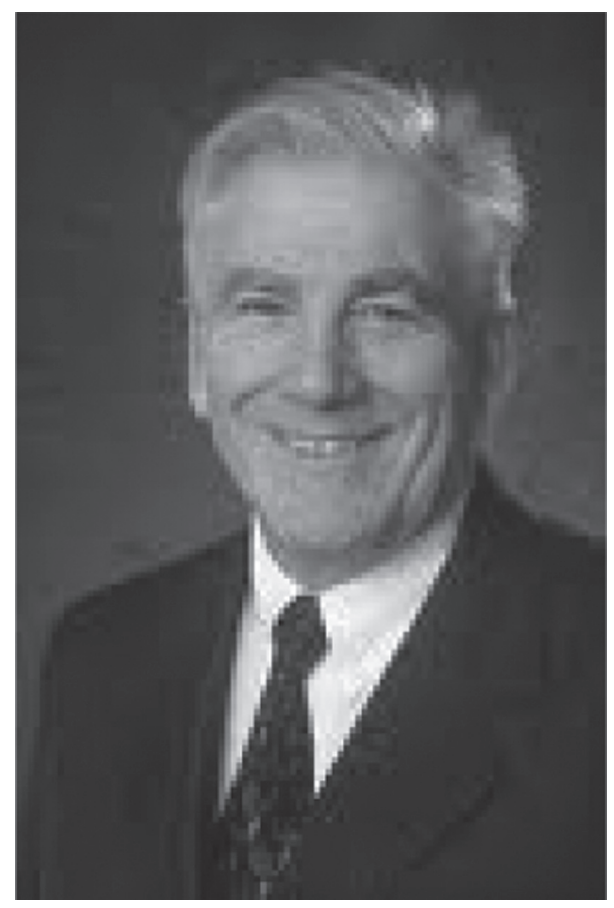

Prof. Howard Morris, PhD, FAACB, FFSc (RCPA) était professeur à l'université de l'Australie Sud et biologiste à SA Pathologie à Adélaide (Australie). Il était l'auteur de plus de 280 publications référencées, ses champs de recherche favoris étaient l'endocrinologie et l'immunologie. Il avait une aura internationale pour ses travaux sur la vitamine D. À ce titre il avait été récompensé par le « Louis Avioli Memorial Lectureship » en 2009.

Comme membre de la Australasian association of clinical biochemists (AACB), il a représenté l'Australie aux assemblées générales de l'IFCC et APFCB entre 1998 et 2004. Il a servi comme membre du conseil AACB (1998-2002) et assuré la fonction de rédacteur en chef du Clinical Biochemist Reviews (1994-2002). Il a reçu de nombreuses récompenses comme : le AACB Outstanding Service Medallion (2003) et le W. Roman Travelling Lectureship (2004). En 2015, il a été récompensé par le ANZBMS career achievement Award. De 1994 à 2004, il a été le rédacteur en chef des Annals of Clinical Biochemistry, dont il a renouvelé la politique éditoriale.

Le Prof. Morris a assuré plusieurs fonctions au sein de l'Asian pacific federation of clinical biochemistry (APFCB) : Chair, IFCC Scientific Committee (2002-2004); Chair, Scientific Organising Committee, et membre du comité d'organisation du 10th Asian Pacific Congress of Clinical Biochemistry (2002-2005). 
Il a exercé comme IFCC Vice-President (2012-2014) ; secrétaire de la division scientifique (2003-2008) ; Chair de la IFCC-International Osteoporosis Foundation, Joint WG on the Standardization of Bone Turnover Markers (2012-2017) et membre de l'IFCC Task Forces on the Global Campaign on Diabetes Mellitus (2003-2008) ainsi que International Clinical Liaison (2009-2011). Il a présidé le comité des IFCC Awards de 2013 à 2014.

Le Prof. Howard Morris a été élu IFCC President en 2016. Il a d'abord servi comme PrésidentElect en 2017, avant de débuter son mandat de trois ans comme Président en janvier 2018. Sa Présidence restera fortement marquée par sa forte énergie et son fort engagement pour les membres de l'IFCC et ses 6 régions. Son souhait était de contribuer à fédérer et à harmoniser le monde de la médecine de laboratoire. Toujours entre deux visites de sociétés membres ou à la recherche de nouveaux partenariats, il n'a jamais décliné une invitation pour promouvoir la Fédération internationale malgré les contraintes de distance à partir de l'Australie. Sa vision du futur de la médecine de laboratoire a souvent inspiré ses collègues du monde entier. Il avait une personnalité attrayante, une attitude positive et un grand sens de l'humour. Toujours à l'écoute de l'autre, il savait convaincre. La Dre Hélène Martin, son épouse était souvent à ses côtés, elle-même scientifique éminente. Elle a toujours été son soutien inconditionnel.

Merci de cette présence inspiratrice. 